VOL. $17(1977), 177-192$.

\title{
Linear monads
}

\section{B.J. Day}

A monad $T=(T, \mu, \eta)$ on a category $C$ is said to be linear with respect to a dense functor $N: A \rightarrow C$ if the operator $T$ is the epimorphic image of a certain colimit of its values on $A$. The main aim of the article is to relate the concept of a linear monad to that of a monad with ronk. A comparison is then made between linear monads and algebraic theories.

\section{Introduction}

In Section 1 we commence with a dense functor $N: A \rightarrow C$ and a monad $T=(T, \mu, \eta)$ on $\mathcal{C}$ such that the canonical transformation $\int^{A} C(N A, C) \cdot T N A+T C$ is an epimorphism. Such a monad is called linear or, more precisely, $N$-linear. We prove that the free algebras on the values $N A$ form a dense full subcategory of the Eilenberg-Moore category $C^{\top}$. The terminology follows that of Day [3], Section 5.

Once the foregoing result is established it allows a comparison to be made between $C^{T}$ as a full subcategory of a functor category $[B, V]$ and the category $C^{t}$ of algebras in $[B, V]$ derived from the resultant algebraic theory of $T$ (ef. Diers [5]). Conditions on $C^{T}$ to be a Birkhoff subcategory of $C^{t}$ are examined in Section 2.

We note here that all categorical algebra is relative to a fixed complete and cocomplete symmetric monoidal closed ground category

Receivec 10 March 1977. The author gratefully acknowledges the support of a Postdoctoral Research Fellowship from the Australian Research Grants Committee. 
$V=(V, V, \otimes, I, \ldots)$ unless otherwise indicated. The terminology and notation are basically derived from Eilenberg and Kelly [6] and Mac Lane [9].

\section{Linear monads and rank}

Throughout this section we suppose that $T=(T, \mu, \eta)$ is a given monad on a category $C$ and that $N: A \rightarrow C$ is a fully faithful dense functor. The standard resolution of $T$ into a Kleisli category and an Eilenberg-Moore category is denoted by

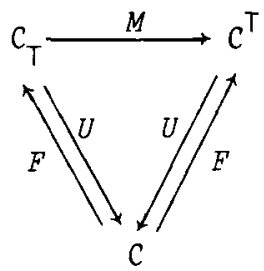

where $M$ is the dense comparison functor. Furthermore, we let $\bar{A}$ denote the full image of $F N: A \rightarrow C_{T}$ and let $\bar{N}: \bar{A} \rightarrow C_{T}$ denote the induced functor such that $F N=\vec{N} F$ :

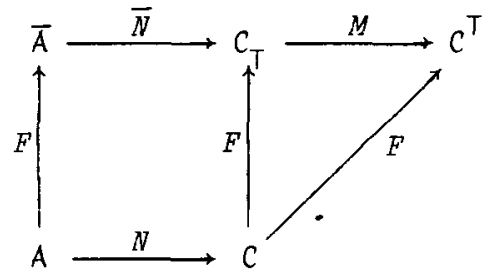

If we now suppose that $A$ is small and $C^{T}$ is cocomplete then, by Day and Kelly [4], (7.1), we have:

LEMMA 1.1. The composite $\overline{M N}: \bar{A} \rightarrow C^{\top}$ is dense iff each natural transformation $\alpha_{B}$ from $C^{\top}(M \overline{N B}, C)$ to $C^{\top}(M \overline{N B}, D)$ is of the form $\mathcal{C}^{\top}(1, f)$ for a unique T-homomorphism $f$ from $C$ to $D . / /$

THEOREM 1.2 (The representation theorem for monads). The compariso: functor $M: C_{T} \rightarrow C^{\top}$ is dense and, for each algebra $(C, \zeta) \in C^{\top}$, the natural transformations from $C^{\top}(M-, C)$ to a prealgebra $G: C_{T}^{o p} \rightarrow V$ 
correspond to the elements in the equaliser of

$$
V G C \underset{V G T \zeta}{\stackrel{V G L}{\longrightarrow}} V G T C,
$$

where $\mu$ and $T \zeta$ are regarded as morphisms in $C_{T}$.

For the proof see Day [2], Proposition 8.2. //

THEOREM 1.3. The composite $\overline{M N}: \bar{A} \rightarrow C^{T}$ is dense if the canonical transformation

$$
\mathcal{C}(T C, D) \rightarrow \int_{A}[\mathrm{C}(N A, C), \mathcal{C}(T N A, D)]
$$

is a monomorphism for all $C \in C$ and $D \in C^{\top}$.

Proof. The notation $U$ will sometimes be omitted. Suppose $\alpha: C^{\top}(F N A, C) \rightarrow C^{\top}(F N A, D)$ is a transformation which is natural in FNA $\in \bar{A}$. An extension $\bar{\alpha}$ is defined by commutativity of

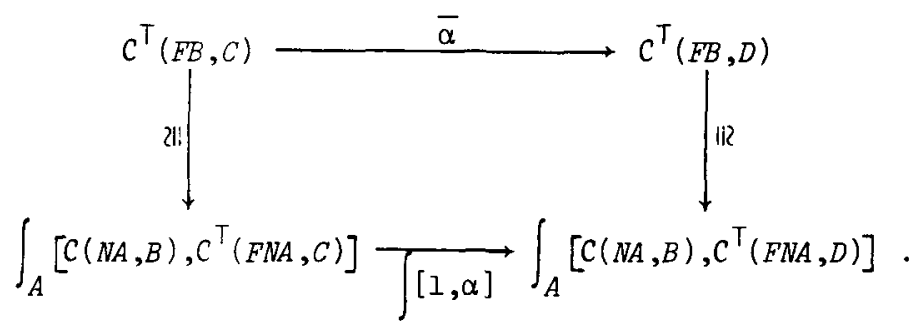

First we note that $\bar{\alpha} \mid \bar{A}=\alpha$ : the diagram

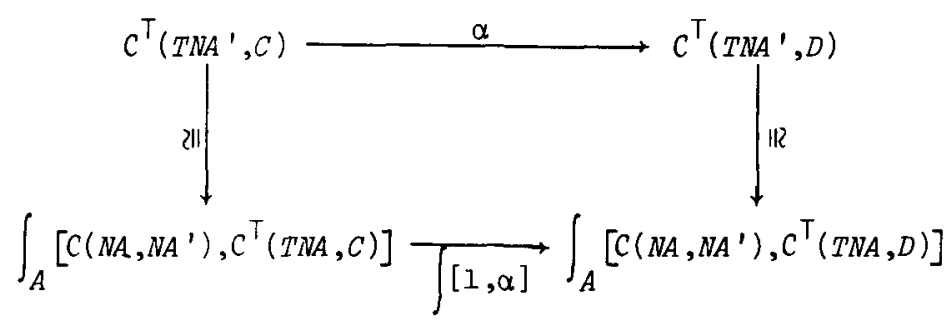

transforms to 


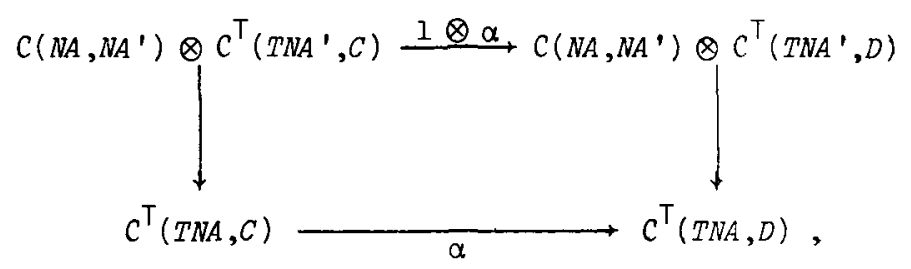

which commutes by naturality of $\alpha$. Secondly, if $\beta$ is a transformation from $C^{\top}(F B, C)$ to $C^{\top}(F B, D)$ which is natural in $E^{\prime} B \in C_{T}$ then $B=\bar{B}$. This follows from the diagram:

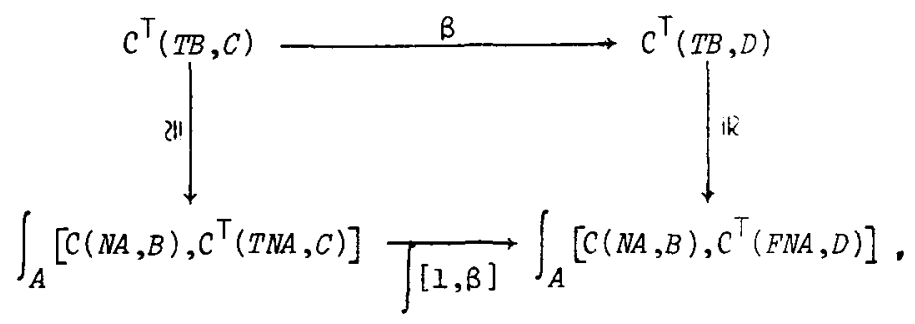

which commutes by naturality of $\beta$. By Theorem 1.2 it is now required to show that $\bar{\alpha}$ corresponds to the element $\bar{\alpha}(\zeta) \in V G C=C_{0}^{T}(F C, D)$ in the equaliser of:

$$
V G C \stackrel{V G \mu}{\stackrel{V G T \zeta}{\longrightarrow}} V G T C
$$

Firstly, because $\bar{\alpha}=\left\{\bar{\alpha}_{F B}\right\}$ is natural in $B \in \mathcal{C}$, we see that the family $\bar{\alpha}_{F B}$ is derived from $\bar{\alpha}_{F C}(\zeta): I \rightarrow C^{\top}(F C, D)$ by the (ordinary) representation theorem:

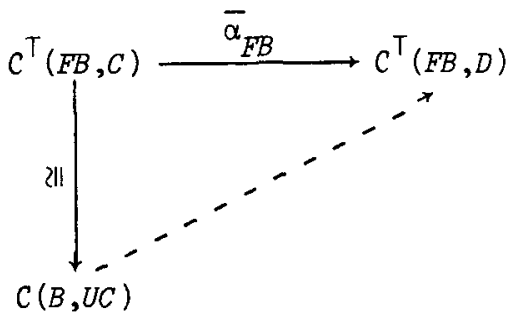

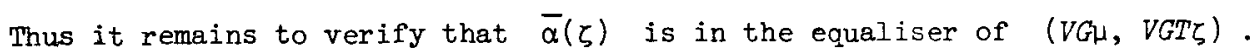
Consider Diagram 1.4; subdiagrams 1 and 4 commute by definition of $\bar{\alpha}$ so 


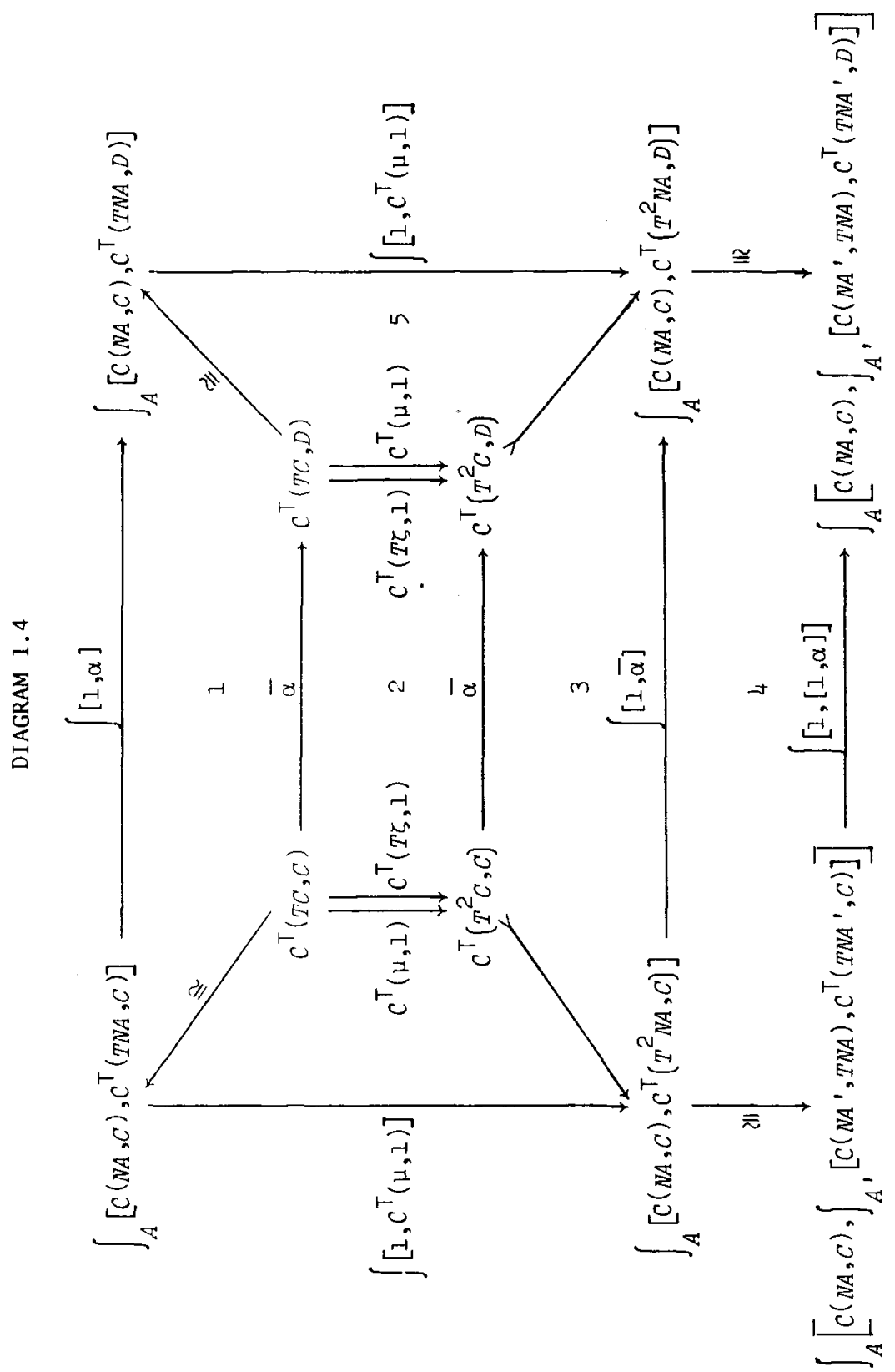


it remains to show that subdiagrams 2, 3, and 5, and the exterior commute. Diagram 2 becomes Diagram 1.5 which clearly commutes. Diagram 3 becomes Diagram 1.6; thus it suffices to show that subdiagram 3' commutes. This follows by applying the representation theorem to $D \in C^{T}$ because both legs are natural in $D$; this diagram then becomes Diagram 1.7.

\section{DIAGRAM 1.5}

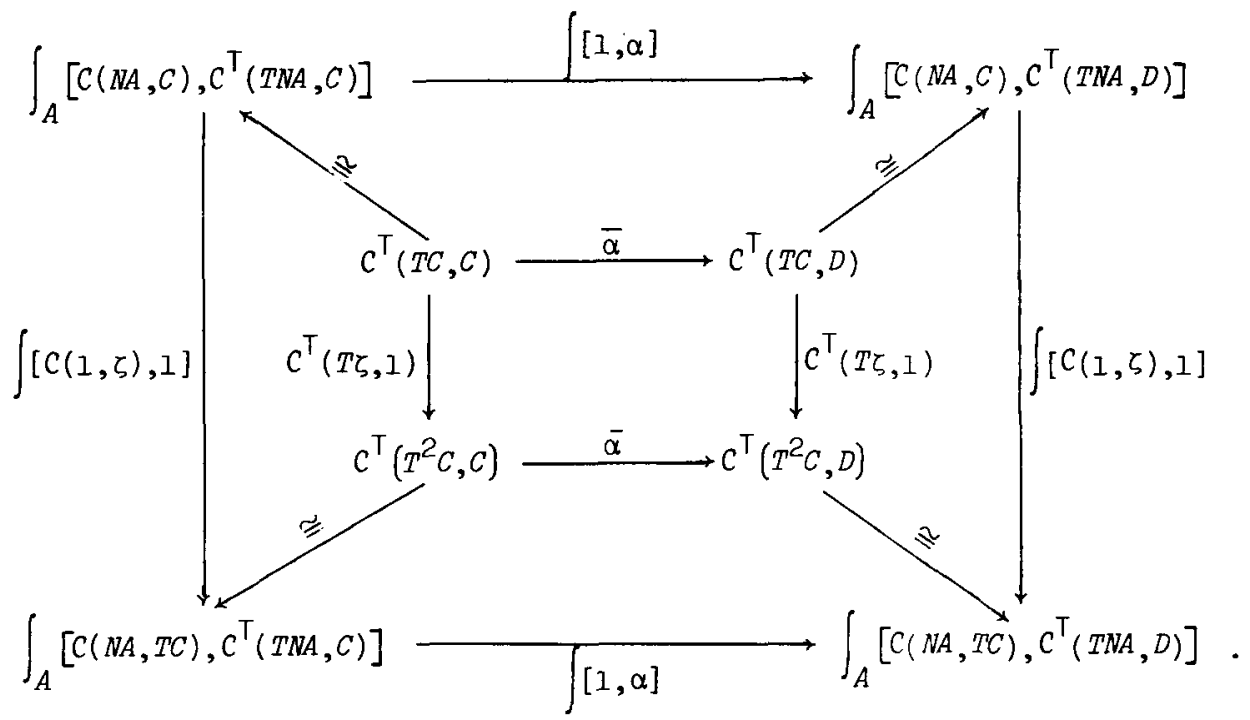




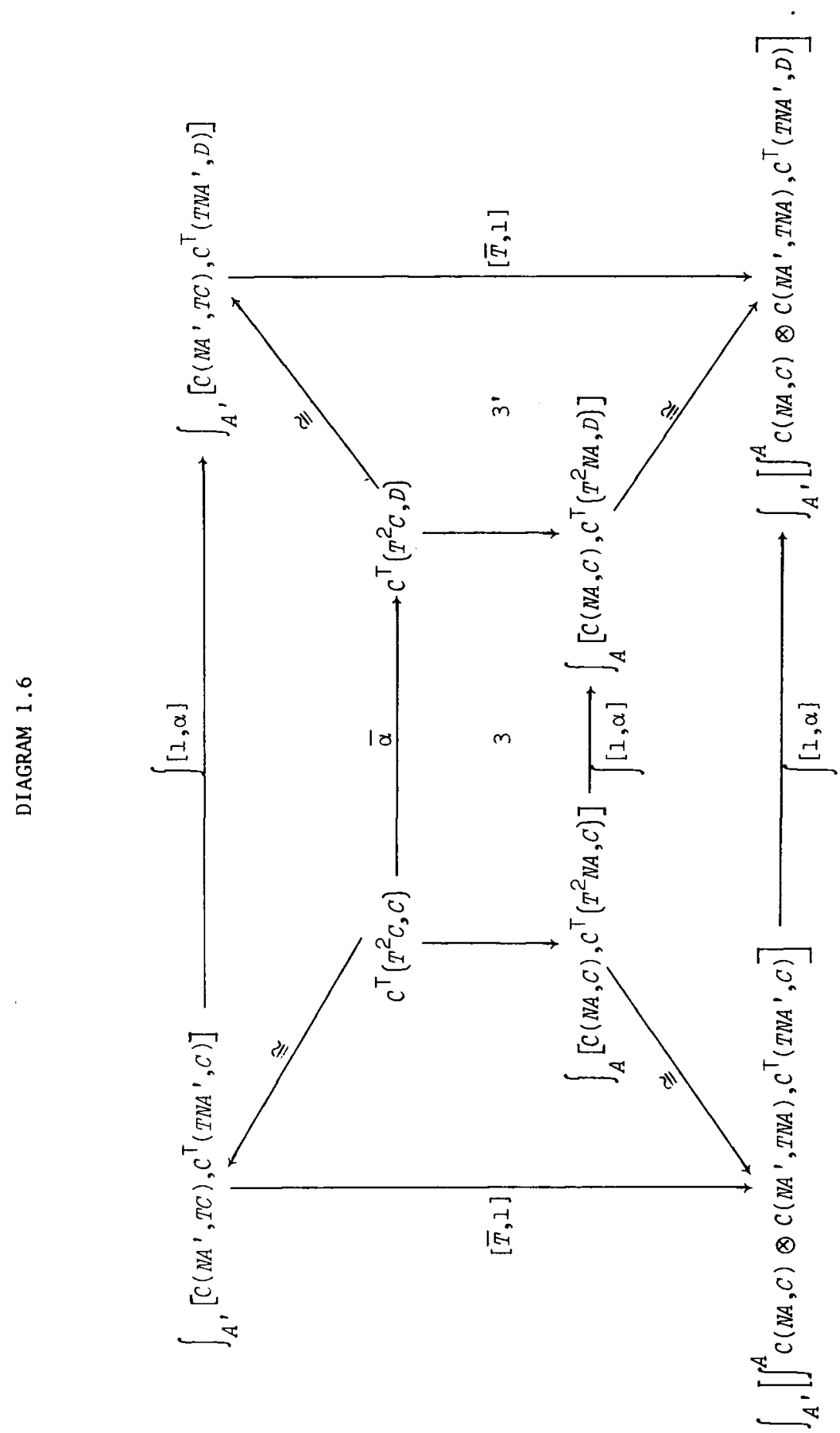


DIAGRAM 1.7

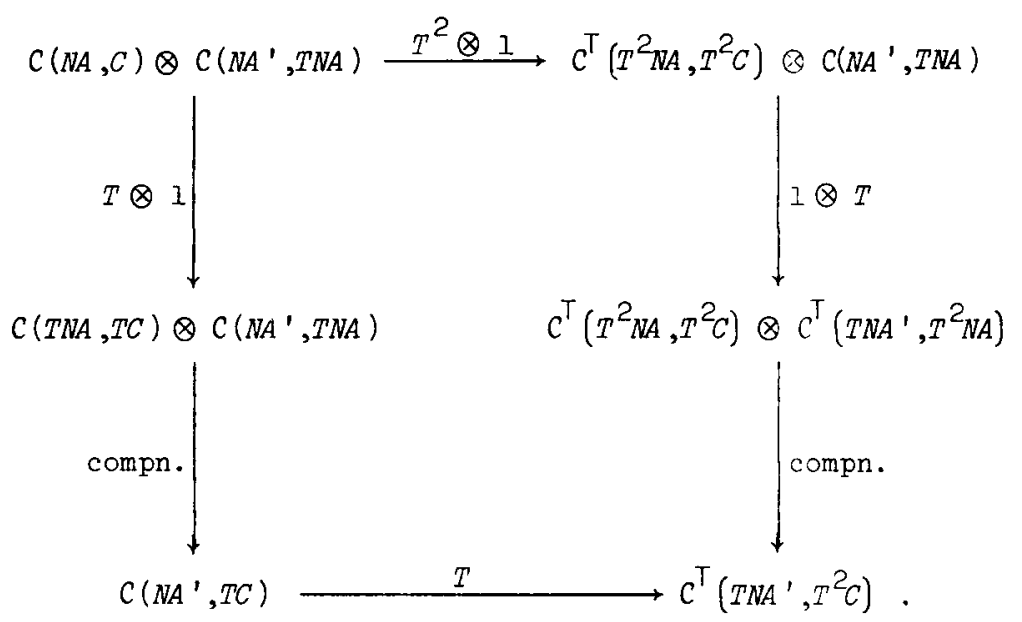

Again, this diagram commutes by the representation theorem applied to $C \in C$. Next consider Diagram 5, which transforms to

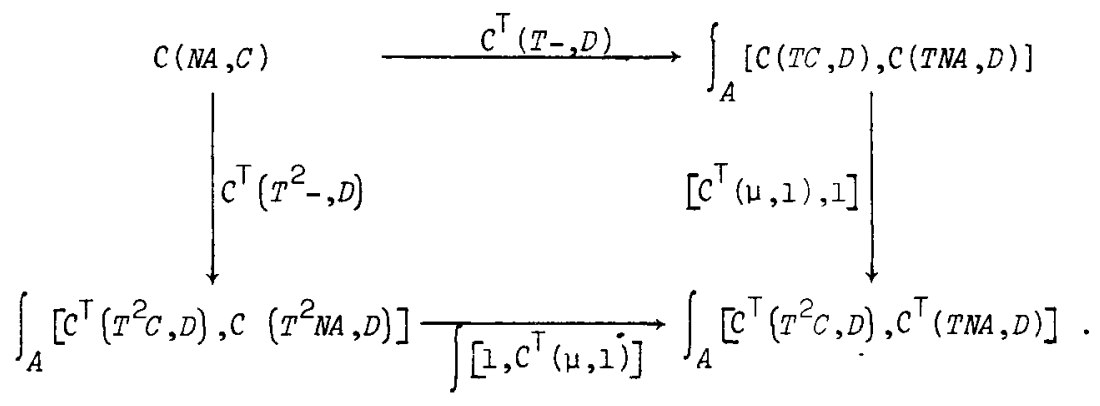

This diagram commutes by naturality of $\mu: T^{2} \rightarrow T$. It remains to check that the diagram

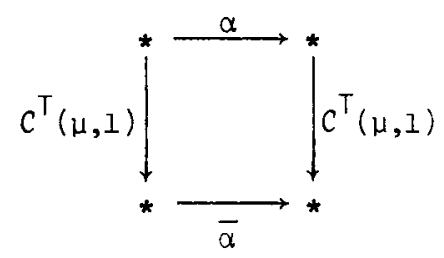

commutes.

This diagram transforms to 


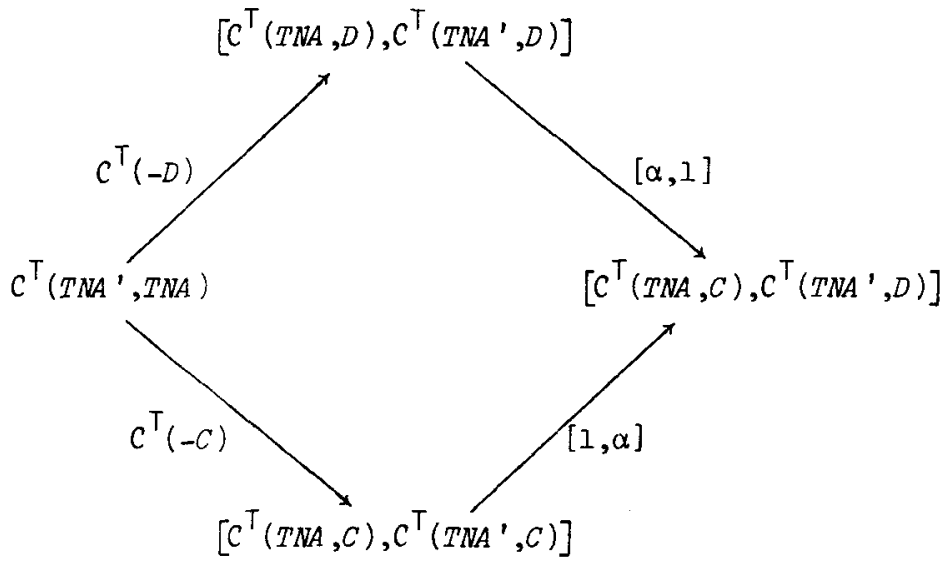

composed with

$$
\mathcal{C}\left(N A^{\prime}, T N A\right) \stackrel{T}{\longrightarrow} C^{\top}\left(T N A^{\prime}, T^{2} N A\right) \stackrel{C^{\top}(I, \mu)}{\longrightarrow} C^{\top}\left(T N A^{\prime}, T N A\right)
$$

thus it commutes by naturality of $\alpha$. //

In view of this result we make the following definition with respect to a fully faithful dense functor $N: A \rightarrow C$.

DEFINITION 1.8. A monad $T=(T, \mu, \eta)$ on $\mathcal{C}$ is called linear (or $N$-linear) if $\mathcal{C}(N A, C) \circ T N A$ exists for all $C \in \mathcal{C}$ and $C(N A, C) \circ T N A \rightarrow T C$ is an epimorphism. The monad is called strictly linear if this transformation is an isomorphism.

COROLLARY 1.9. If $\mathrm{T}$ on $\mathrm{C}$ is $N$-linear then the canonical diagram

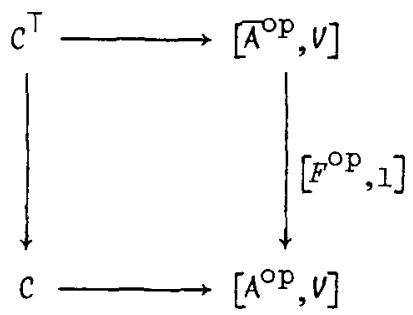

commutes (to within a natural isomorphism) where the horizontal functors are fulzy faithfuz. - //

\section{Comparison with algebraic theories}

Let $N: A \rightarrow C$ and $T=(T, \mu, \eta)$ be as in Section 1 . Then 
$t=F^{\circ p}: A^{\circ p} \rightarrow \bar{A}^{\circ p}=T$ is an $N$-algebraic theory in the sense of Diers [5]. Thus we form the category $C^{t}$ of $t$-algebras by means of the pullback

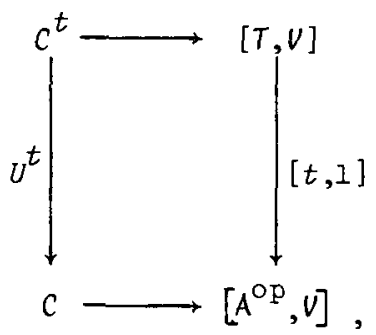

where the horizontal functors are fully faithful.

By Corollary 1.9, $C^{\top}$ is a full reflective subcategory of $[T, V]$ and it lies in $\mathrm{c}^{t}$. This gives a reflection $s: \mathrm{C}^{t} \rightarrow \mathrm{C}^{\top}$ :

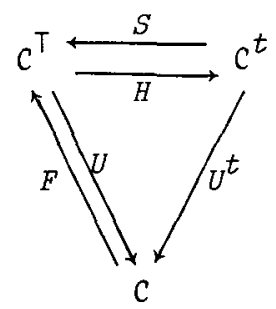

THEOREM 2.1. If $T$ is strictly linear then $C^{\top}$ is category equivalent to $C^{t}$.

Proof. Because $C(N A, C) \circ T N A \cong T C$ we have that $T$ preserves $N$-absolute colimits. Thus the hypotheses of Diers [5], Theorem 5.1, are satisfied by $F \dashv U$. //

Now suppose that $C$ has canonical factorisations for the system \{strong epimorphisms and monomorphisms\} (ef. Freyd and Kelly [7]).

PROPOSITION 2.2. If the transformation

$$
\int^{A} \mathcal{C}(N A, C) \otimes \mathcal{C}\left(N A^{\prime}, T N A\right) \rightarrow \mathcal{C}\left(N A^{\prime}, T C\right)
$$

is a strong epimorphism in $V$ and $C(N A, C) \circ$ TNA exists in $C$ and $C(N A,-): C \rightarrow V$ preserves strong epimorphisms for all $A \in A$, then the 
wnit $n$ of the reflection $S \rightarrow H$ is a strong epimorphism.

Proof. On applying - o $N A^{\prime}$ to both sides of (2.1) we see that $C(N A, C) \circ T N A \rightarrow T C$ is a strong epimorphism. Thus Theorem 1.3 applies and also $T$ preserves strong epimorphisms since, if $e: C \rightarrow D$ is a strong epimorphism in $C$, we have that

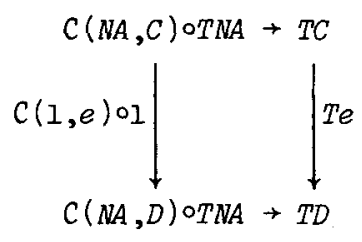

commutes. Thus the diagonal is a strong epimorphism so $T$ te is a strong epimorphism. Now consider the factorisation $n_{C}: C \rightarrow D \longleftrightarrow S C$ in $C$. It is required to show that $D$ has a T-algebra structure. This structure is derived from the following diagram:

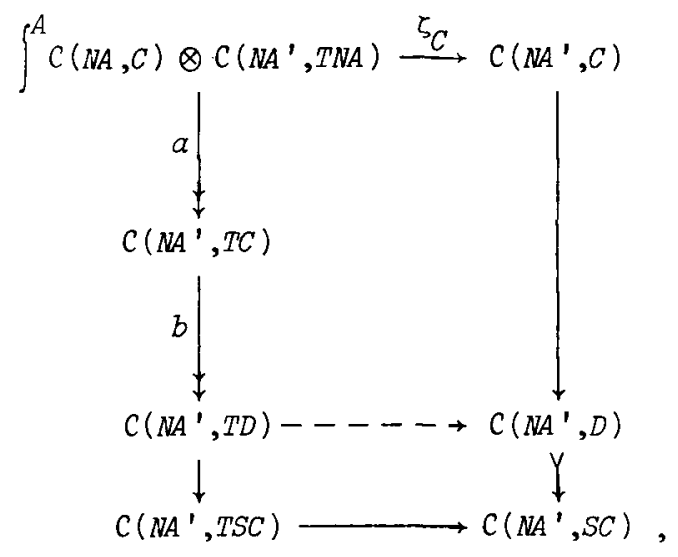

where $a$ and $b$ are both strong epimorphisms and the top morphism ${ }^{\zeta} C$ is derived from the $C^{t}$-structure on $C$ in the following manner. An algebra $(C, \zeta) \in \mathcal{C}^{t}$ comprises $C \in \mathcal{C}$ together with

$$
\zeta_{C}: \int^{A} C\left(N A^{\prime}, T N A\right) \otimes C(N A, C) \rightarrow \mathcal{C}\left(N A^{\prime}, C\right)
$$

that is, a structure for the monad $[t, 1] \bar{t}$ on $\left[A^{\circ p}, V\right]$ where $\bar{t} \rightarrow[t, 1]$. Then, by factorisation, the dashed arrow provides a T-algebra structure on $D$, using the density of $N$. // 
PROPOSITION 2.3. Suppose the unit $n$ of the reflection $S \rightarrow B$ is a strong epimorphism and $\mathcal{C}$ has kernel pairs. If $U: \mathcal{C}^{\top} \rightarrow \mathcal{C}$ reflects kernel pairs then $C^{\top}$ is closed in $c^{t}$ under coequalisers.

Proof. Both $U$ and $U^{t}$ create kernel pairs and we omit them from the notation. Let $q: H C \rightarrow D$ be a coequaliser in $C^{t}$ and let $p=n_{D} \cdot q$. Let $\left(\pi_{1}, \pi_{2}\right)$ be the kernel pair of $q$ in $C^{t}$ and let $\left(\phi_{1}, \phi_{2}\right)$ be the kernel pair of $p$ in $C^{\top}$. This gives

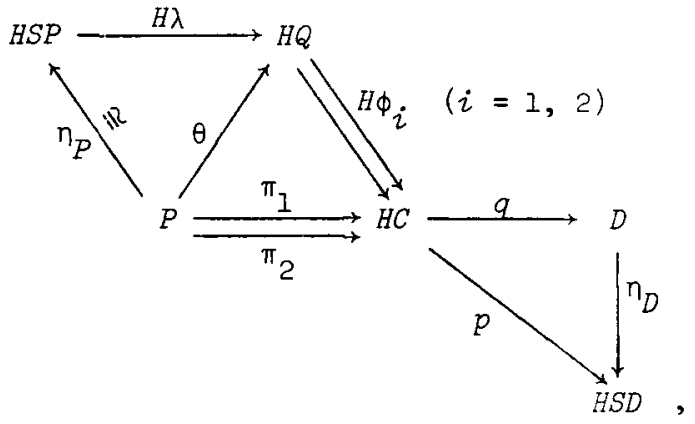

where $\theta$ is monic, so $\eta_{p}$ is monic and thus is an isomorphism. This implies that $p$ is the coequaliser of $\left(H \phi_{1} . H \lambda, H \phi_{2} . H \lambda\right)$ in $c^{\top}$ and that this latter pair is a kernel pair in $c^{t}$. Thus $\left(\phi_{1} \lambda, \phi_{2} \lambda\right)$ is a kernel pair in $\mathcal{C}^{\top}$, so $\lambda$ is an isomorphism, so $\theta$ is an isomorphism, so $\eta_{D}$ is an isomorphism, as required to show that $D$ lies in $\mathrm{C}^{\top} . / /$

$$
\text { COROLLARY 2.4. If } \int^{A} \mathcal{C}(N A, C) \otimes \mathcal{C}\left(N A^{\prime}, T N A\right) \rightarrow \mathcal{C}\left(N A^{\prime}, T C\right) \text { is a }
$$
strong epimorphism in $V$ and $C(N A, C) \circ T N A$ exists in $C$ and $C(N A,-)$ presemes strong epimorphisms for all $A \in A$ and $C$ has kemel pairs reflected by $U: C^{\top} \rightarrow \mathcal{C}$, then $C^{\top}$ is a Birkhoff reflective subcategory of $c^{t} \cdot 1 /$

PROPOSITION 2.5. If $\mathrm{c}^{t}$ is cocomplete and $\mathrm{C}(N A,-)$ preserves coequalisers of reflective pairs, then $c^{t}$ is monadic over $c$ iff 
(a) $f$ is a coequaliser in $c^{t}$ iff $U^{t} f$ is a coequaliser in $c$, and

(b) $U^{t}$ reflects kemel pairs.

Proof. If $C^{t}$ is cocomplete then $F^{t} \rightarrow U^{t}$ exists and $U^{t} F^{t}$ preserves coequalisers of reflective pairs since $U^{t}: C^{t} \rightarrow C$ creates coequalisers because $C\left(N A^{\prime},-\right)$ preserves them (cf. Diers [5], Proposition 1.1). Thus the result follows from Borceux and Day [1], Corollary 6.2. //

PROPOSITION 2.6. Suppose $\mathrm{C}$ and $\mathrm{C}^{t}$ are cocomplete and let $K:[T, V] \rightarrow C^{t}$ denote the canonical reflection. If $U^{t}$ preserves epimorphisms and those unit components of the form $T(t A,-) \rightarrow K T(t A,-)$ are epimorphisms, then $U^{t} F^{t}$ generates a linear monad.

Proof. We have

$$
U^{t} F^{t} N A=U^{t} K\left(\int^{A^{\prime}} c\left(N A^{\prime}, N A\right) \otimes T\left(t A^{\prime},-\right)\right) \cong U^{t} K(T(t A,-))
$$

by the representation theorem because $N$ is fully faithful. Also

$$
U^{t}{ }^{t} C=U^{t} K\left\{\int^{A} C(N A, C) \otimes T(t A,-)\right\} .
$$

Thus, to show that

$$
\int^{A} C(N A, C) \cdot U^{t_{F}} t_{N A} \rightarrow U^{t_{F} t_{C}}
$$

is an epimorphism consider the following diagram

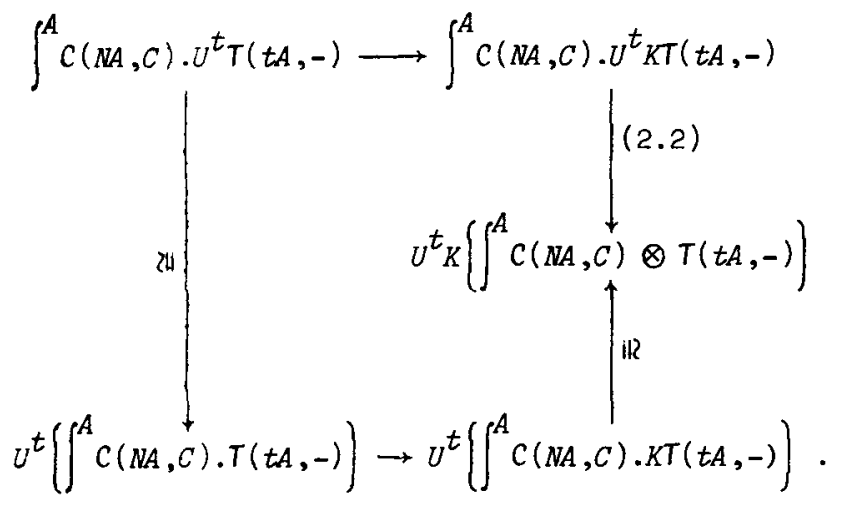


The bottom arrow is an epimorphism by hypothesis, so (2.2) is an epimorphism, as required. //

\section{Example}

Suppose the ground category $V$ has canonical $E$ - $M$ factorisations for the system $E-M=$ \{strong epimorphisms and monomorphisms\} (see Freyd and Kelly [7]). Suppose also that $V$ has arbitrary cointersections of E-quotients and that finite powers preserve strong epimorphisms.

DEFINITION 3.1. A functor $G: A \rightarrow V$ from a category $A$ with finite products to $V$ is said to E-preseme finite products if the canonical morphism $G\left(A \times A^{\prime}\right) \rightarrow G A \times G A^{\prime}$ is a strong epimorphism for all $A, A^{\prime} \in A$.

DEFINITION 3.2. Let $M: A \rightarrow B$ be a functor between categories with finite products. Then $V$ is said to satisfy axiom $E(\pi)$ if the left Kan extension of a functor $G: A \rightarrow V$ which $E$-preserves finite products along $M$ again $E$-preserves finite products.

One then obtains results precisely analogous to those obtained for axiom $\pi$ in Borceux and Day [1], Sections 1 and 2.

DEFINITION 3.3. If $T$ is a finitary algebraic theory (see Borceux and Day [1], Definition 3.I) then a functor $G: T \rightarrow V$ which $E$-preserves finite products is called an E-algebra (of $T$ ).

Now let $T^{q}$ denote the category of 'E-algebras for $T$, regarded as a full subcategory of $[T, V]$. Let $T^{b}$ denote the ordinary category of algebras of $T$; namely, the full subcategory of $[T, V]$ defined by the finite-product-preserving functors. Then there are inclusions $T^{b} \subset T^{q} \subset[T, V]$. The second embedding is coreflective and the coreflection maps $G$ to the union of the E-algebras which are $M$-subfunctors of $G$; the coreflection counit lies in $M$. The first embedding is reflective and the reflection maps $A \in T^{q}$ to the largest $T^{b}$ $E$-quotient of $A$; the reflection unit is in $E$. Thus we have 


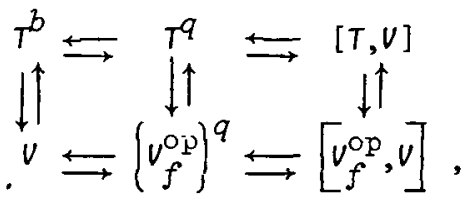

where $v_{f}$ is the initial finitary theory and where the centre adjunction is a strictly linear monadic situation.

THEOREM 3.4. If $V$ satisfies the hypotheses of this section (and satisfies axiom $E(\pi)$, then a monad $T$ on $V$ generates a Birkhoff subcategory of an algebraic category $T^{b}$ iff

(a) $V^{\top}$ has coequalisers,

(b) $\int^{m}[m, X] \otimes[n, T m]+[n, T X]$ is a strong epimorphism, and

(c) $U: V^{\top} \rightarrow U$ reflects kermel pairs.

Proof. Because $U^{\top}$ has coequalisers iff $U^{\top}$ is cocomplete (see Linton [8]) the conditions are sufficient by Corollary 2.4. Necessity of (a) is clear since $T^{b}$ is always cocomplete. Moreover, if $V^{\top}$ is a Birkhoff subcategory of $T^{b}$ then the unit of the composite reflection $T^{q} \rightarrow T^{b} \rightarrow V^{T}$ is a strong epimorphism. This implies that $(b)$ is necessary. Finally, the functor $U^{t}: T^{b} \rightarrow V$ reflects kernel pairs and the Birkhoff property implies that the embedding $V^{\top} \subset T^{b}$ reflects kernel pairs, so (c) is necessary. $/ /$

An example of a monad which satisfies $(a)$ and $(b)$ but not $(c)$ is the reflection to Hausdorff $k$-spaces from non-Hausdorff $k$-spaces.

\section{References}

[1] Francis Borceux and Brian Day, "Universal algebra in a closed category", J. Pure Appl. AZgebra (to appear). 
[2] Brian Day, "On closed categories of functors II", Category seminar, 20-54 (Proc. Sydney Category Seminar 1972/1973. Lecture Notes in Mathematics, 420. Springer-Verlag, Berlin, Heidelberg, New York, 1974 ).

[3] B.J. Day, "Density presentations of functors", BulZ. Austral. Math. Soc. 16 (1977), $427-448$.

[4] B.J. Day and G.M. Kelly, "Enriched functor categories", Report of the Midwest Category Seminar III, 178-191 (Lecture Notes in Mathematics, 106 . Springer-Verlag, Berlin, Heidelberg, New York, 1969).

[5] Y. Diers, "Foncteur pleinement fidèle dense classant les algèbres", (Publications Internes de I'U.E.R. de Mathématiques Pures et Appliquées, 58. Université des Science et Techniques de Lille I, 1975).

[6] Samuel Eilenberg and G. Max Kelly, "Closed categories", Proc. Conf. Categorical Algebra, La Jolla, California, 1965, 421-562 (Springer-Verlag, Berlin, Heidelberg, New York, 1966).

[7] P.J. Freyd and G.M. Kelly, "Categories of continuous functors, I", $J$. Pure Appl. Algebra 2 (1972), 169-191.

[8] F.E.J. Linton, "Coequalisers in categories of algebras", Seminar on Triples and Categorical Homology Theory, 75-90 (Lecture Notes in Mathematics, 80. Springer-Verlag, Berlin, Heidelberg, New York, 1969).

[9] S. Mac Lane, Categories for the working mathematician (Graduate Texts in Mathematics, 5. Springer-Verlag, New York, Heidelberg, Beriin, 1971).

Department of Pure Mathematics, University of Sydney, Sydney, New South Wales. 